\title{
Analysis of the agglutinating activity from unicellular algae
}

\author{
Chun-Yao Chu $\cdot$ Rang Huang $\cdot$ Lian-Ping Lin
}

Received: 22 February 2006 / Accepted: 26 October 2006 / Published online: 10 February 2007

(C) Springer Science + Business Media B.V. 2007

\begin{abstract}
Agglutinating activity often varies both between and within the algal species assayed. However, it is difficulty to interpret such variation without further analysis. We report a statistical analysis of agglutinating activities against human, cow, sheep, and pig erythrocytes, using cell extracts from 43 taxa (strains) of freshwater microalgae. Most of the extracts agglutinated erythrocytes from at least one of the sources, but pig erythrocytes appeared to be most suitable for the detection of agglutination reactions. Chlorella cell extracts preferentially agglutinated human erythrocytes, whereas extracts of other taxa were less active against mammalian erythrocytes. Cluster analysis generated four distinct subclusters of taxa, characterized by different specificities for antigens or carbohydrate receptors on the erythrocytes. Principal component analysis further separated the agglutination characteristics of Chlamydomonas from Chlorella on the first two components. Specificity for pig erythrocytes accounted for most of the clustering or grouping of algal taxa in multivariate analysis. However, clustering or grouping patterns of Chlorella species on haemagglutinating activity resembled that based on DNA sequences, revealing a possible genetic connection of agglutinins and their biochemical characteristics in algal cells. Variability of agglutination reactions among the algae investigated is simplified and interpreted most easily using multivariate analysis.
\end{abstract}

\section{C.-Y. Chu • L.-P. Lin}

Institute of Microbiology and Biochemistry,

National Taiwan University,

Taipei 106, Taiwan, R.O.C.

\section{R. Huang $(\bowtie)$}

Institute of Oceanography, National Taiwan University,

Taipei 106, Taiwan, R.O.C.

e-mail: sping.bbs@yahoo.com.tw
Key words agglutinating activity $\cdot$ microalgae $\cdot$ cluster analysis $\cdot$ principal component analysis

\section{Introduction}

Agglutinins are widely distributed in nature. They bind to specific carbohydrates and produce unique effects such as the aggregation of cells or the precipitation of glycoconjugates. Agglutinins can thus serve as probes of carbohydrates and are useful tools in cell biology, immunology, in the study and treatment of cancers, and in a range of biochemical and biomedical research areas.

Since the first report by Boyd et al. (1966), many researchers have investigated the agglutinating capacities of marine algal extracts (Blunden et al. 1978; Rogers et al. 1980; Hori et al. 1981; Fabregas et al. 1985; Freitas et al. 1997). For the microalgae, agglutinating activity has been reported for Lyngbya majuscula (Boyd et al. 1966) and Chlamydomonas spp. (Brown et al. 1968; Saito and Matsuda 1984). However, our knowledge of microalgal agglutinins and their agglutination characteristics is limited. Agglutinins from Anabaena flosaquae and Microcystis aeruginosa agglutinated mouse erythrocytes (Carmichael and Bent 1981), and Raphidophyceae, Bacillariophyceae species, and culture media conditioned by Alexandrium cohorticula and Chattonella antiqua caused agglutination (Hori et al. 1996). Some special characteristics of microalgal agglutinins have been reported, for example, that aerobic growth conditions could inhibit agglutinin production in Microcystis viridis (Yamaguchi et al. 1999), and that M. aeruginosa exhibited calcium-dependent agglutinating activity (Sakamoto et al. 1996). Microcystis aeruginosa and Oscillatoria agardhii agglutinins have unique properties 
that are not commonly found in higher plants (Yamaguchi et al. 1998; Sato et al. 2000).

In assaying for agglutination, sheep and rabbit erythrocytes are more sensitive than human erythrocytes in the detection of agglutinins from seaweeds (Hori et al. 1981; Fabregas et al. 1985; Freitas et al. 1997). However, monkey and human erythrocytes are more susceptible than sheep erythrocytes to the marine unicellular algal extracts in our previous studies (Liao and Huang 2000; Liao et al. 2001). Thus, microalgal agglutinins have diverse specificity for carbohydrate or antigens, as shown by seaweeds.

Freshwater microalgae have the advantage that they can be cultivated on a large scale at extremely high growth rates. These organisms thus form potentially important sources of novel agglutinins for biomedical research and commercial applications. Agglutinins have been identified in various types of cells and tissues of all taxonomies to higher vertebrates (Blunden et al. 1978; Carmichael and Bent 1981; Fabregas et al. 1985). Some reports show that agglutinin is more common in marine algae (Rogers et al. 1980), and agglutination assays with numerous marine algae indicated that agglutinins and their activities had taxonomic significance (Rogers and Fish 1991). Thus, genetic connection of haemagglutinating activity used on taxonomic significances is an interesting subject.

Because agglutinating activity varies greatly between and within the species assayed (Rogers and Fish 1991), it is often difficult to interpret such variations when large numbers of taxa are screened. In order to understand the relationship between the unicellular algal agglutinating activity and taxonomic significance, we analyzed the variability of agglutinating activity in freshwater microalgae using multivariate analysis. Additionally, the analysis results were used for comparison with an analysis based on DNA sequences.

\section{Materials and methods}

Forty-three strains of freshwater microalgae, mostly belonging to Chlorella and Chlamydomonas, were used in this study (Table 1). Most were obtained from Culture Collections such as ATCC (American Type Culture Collection), CCAP (Culture Collection of Algae and Protozoa, CCMP (The Provasoli-Guillard Center for Culture of Marine Phytoplankton) and IAM (IAM Culture Collection, Japan). Chlorella sp. I, Chlorella sp. 21, Chlorella sp. W, and C. pyrenoidosa were collected from rivers of Taiwan and isolated in our laboratory, while Selenastrum capricornutum, Monoraphidium contorfum, Coslastrum astroideum, Eutetramorus fottii, Scenedesmus quadricauda, and $S$. ecornis were kindly provided by Professor J.-T. Wu, Institute of Botany, Academia Sinica,
Taiwan, R.O.C. The algae were grown and maintained in proteose medium (Illman et al. 2000) at $25^{\circ} \mathrm{C}$ at an irrandiance of $80 \mu \mathrm{mol}$ photons $\mathrm{m}^{-2} \mathrm{~s}^{-1}$ with a $12: 12 \mathrm{~h}$ light:dark cycle. Algal cells were harvested at the stationary growth phase and concentrated by centrifugation. The harvested cells were lyophilized and kept at $-20^{\circ} \mathrm{C}$ until used for extraction.

\section{Preparation of cell extracts and erythrocytes}

The harvested cells were freeze-dried and ground into powder with liquid nitrogen. Cell powder was extracted at a $1: 10$ ratio (w:v) in $20 \%$ ethanol at $4{ }^{\circ} \mathrm{C}$ overnight. Extracts were centrifuged and the supernatants collected for agglutination assays. Human, cow, sheep, and pig erythrocytes were used for the agglutination assay. Fresh human type A, $\mathrm{B}$ and $\mathrm{O}$ blood was provided by Union Clinical Laboratory, Taipei, Taiwan, and cow, sheep, and pig erythrocytes were from the Agricultural Experimental Farm, Department of Animal Science, National Taiwan University. Erythrocytes were concentrated and washed three times in phosphate buffer saline (PBS) by centrifugation, then diluted to a final concentration of $2 \%(\mathrm{v} / \mathrm{v})$ in PBS.

\section{Haemagglutination assay}

Agglutinating activity was assayed according to Hori et al. (1981). An aliquot of algal extract $(50 \mu \mathrm{L})$ was serially diluted two-fold with PBS in the wells of microtitre plates. An equal volume of erythrocyte suspension was then added to the well and mixed with the algal extract at room temperature for $2 \mathrm{~h}$. The control used in this assay was aqueous ethanol without extract. Activity was expressed as a titre based on the reciprocal of the highest two-fold dilution that produced positive agglutination.

\section{Data analysis}

We used various statistical and multivariate techniques to interpret the variability in haemagglutination activity. Firstly, simple correlation coefficients were calculated, based on the haemagglutination reactions of each of the 43 cell extracts, paired with each erythrocyte type. This was followed by multivariate analysis. To reduce the bias caused by any absence of haemagglutination in the analyses, titres were $\log (\mathrm{x}+1)$ transformed. Cluster analysis was carried out with complete linkage based on the similarity matrix of algal haemagglutination activities, using Primer 5 software (Primer-E, Plymouth, UK). Multivariate principal component analysis also using Primer 5 was used to interpret agglutination variability. In this analysis, all relationships among variables (sources of erythrocytes) were accounted for by the relatively independent 
Table 1 Hemagglutinating activity of cell extracts from the study microalgae for different types of native erythrocytes; activity is expressed in titer. The numbers in parentheses are the serial numbers used in the Figs. 1 and 2

\begin{tabular}{|c|c|c|c|c|c|c|}
\hline \multirow[t]{3}{*}{ Algal species } & \multicolumn{6}{|c|}{ Erythrocyte } \\
\hline & \multicolumn{3}{|c|}{ Human } & \multirow[t]{2}{*}{ Cow } & \multirow[t]{2}{*}{ Sheep } & \multirow[t]{2}{*}{ Pig } \\
\hline & A & $\mathrm{B}$ & $\mathrm{O}$ & & & \\
\hline \multicolumn{7}{|l|}{ Cyanophyceae } \\
\hline (1) Spirulina maxima ATCC 53871 & $2^{3}$ & $2^{3}$ & $2^{3}$ & 0 & $2^{3}$ & $2^{3}$ \\
\hline (2) S. platensis ATCC 53844 & $2^{3}$ & $2^{3}$ & $2^{3}$ & $2^{3}$ & $2^{2}$ & $2^{3}$ \\
\hline (3) S. subsalsa IAM 223 & 0 & 0 & 0 & 0 & $2^{3}$ & 0 \\
\hline (4) Synechococcus leopoliensis IAM M6 & 0 & 0 & 0 & 0 & $2^{3}$ & $2^{5}$ \\
\hline \multicolumn{7}{|l|}{ Chlorophyceae } \\
\hline (5) Chlorella sp.I & $2^{6}$ & $2^{8}$ & $2^{6}$ & 0 & 0 & 0 \\
\hline (6) Chlorella sp. 21 & $2^{5}$ & $2^{6}$ & $2^{8}$ & $2^{7}$ & $2^{8}$ & $2^{7}$ \\
\hline (7) Chlorella sp.W & $2^{3}$ & $2^{2}$ & $2^{4}$ & 0 & 0 & $2^{3}$ \\
\hline (8) C. ellipsoidea IAM87 & $2^{10}$ & $2^{11}$ & $2^{10}$ & $2^{4}$ & $2^{4}$ & $2^{5}$ \\
\hline (9) C. fusca var. fusca IAM 101 & $2^{5}$ & $2^{6}$ & $2^{5}$ & 0 & 0 & 0 \\
\hline (10)C. fusca var. vacuolata IAM 28 & $2^{4}$ & $2^{6}$ & $2^{5}$ & 0 & 0 & 0 \\
\hline (11) C. kessleri IAM 208 & $2^{4}$ & $2^{5}$ & $2^{4}$ & 0 & 0 & 0 \\
\hline (12) C. luteovirtidis CCAP 213/9 & $2^{6}$ & $2^{8}$ & $2^{5}$ & $2^{4}$ & $2^{2}$ & $2^{7}$ \\
\hline (13) C. protothecoides IAM 150 & $2^{9}$ & $2^{11}$ & $2^{9}$ & 0 & 0 & 0 \\
\hline (14) C. protothecoides var. mannophila IAM 206 & $2^{5}$ & $2^{6}$ & $2^{5}$ & 0 & 0 & $2^{6}$ \\
\hline (15) C. pyrenoidosa & $2^{9}$ & $2^{11}$ & $2^{10}$ & $2^{5}$ & $2^{4}$ & $2^{6}$ \\
\hline (16) C. saccharophila IAM 212 & $2^{5}$ & $2^{4}$ & $2^{3}$ & 0 & 0 & $2^{5}$ \\
\hline (17) C. saccharophila var. ellipsidea ATCC 30404 & $2^{3}$ & $2^{4}$ & $2^{6}$ & 0 & 0 & 0 \\
\hline (18) C. sorokiniana IAM 212 & $2^{7}$ & $2^{4}$ & $2^{3}$ & 0 & $2^{9}$ & $2^{6}$ \\
\hline (19) C. vulgaris CCAP 211/13 & $2^{7}$ & $2^{7}$ & $2^{7}$ & $2^{8}$ & $2^{6}$ & $2^{8}$ \\
\hline (20) C. vulgaris NIES 227 & $2^{5}$ & $2^{3}$ & $2^{3}$ & $2^{4}$ & $2^{4}$ & $2^{5}$ \\
\hline (21) C. xanthella ATCC 30411 & $2^{5}$ & $2^{7}$ & $2^{5}$ & 0 & 0 & $2^{8}$ \\
\hline (22) C. zofingiensis IAM 111 & $2^{8}$ & $2^{9}$ & $2^{8}$ & 0 & $2^{3}$ & $2^{9}$ \\
\hline (23) Chlamydomonas applanataIAM 237 & 0 & 0 & 0 & 0 & 0 & 0 \\
\hline (24) C. asymmetrica IAM 234 & $2^{5}$ & $2^{4}$ & $2^{5}$ & 0 & $2^{2}$ & $2^{5}$ \\
\hline (25) C. debaryana IAM 215 & 0 & 0 & 0 & 0 & 0 & 0 \\
\hline (26) C. fimbriata IAM 230 & 0 & 0 & $2^{2}$ & 0 & 0 & 0 \\
\hline (27) C. leiostraca IAM 284 & 0 & 0 & 0 & 0 & 0 & 0 \\
\hline (28) C. noctigama IAM 557 & 0 & 0 & 0 & $2^{2}$ & $2^{4}$ & $2^{5}$ \\
\hline (29) C. oblonga IAM 265 & 0 & 0 & 0 & 0 & 0 & 0 \\
\hline (30) C. proteus IAM 560 & 0 & 0 & 0 & 0 & 0 & 0 \\
\hline (31) C. pulvinata IAM 267 & $2^{3}$ & $2^{3}$ & $2^{3}$ & 0 & $2^{2}$ & $2^{5}$ \\
\hline (32) C. reinhardtii IAM 238 & 0 & 0 & 0 & 0 & 0 & 0 \\
\hline (33) C. segnis IAM 254 & 0 & 0 & 0 & 0 & 0 & 0 \\
\hline (34) C. simplex IAM 281 & 0 & 0 & 0 & 0 & 0 & 0 \\
\hline (35) C. subangulosa IAM 563 & 0 & 0 & 0 & 0 & 0 & 0 \\
\hline (36) C. ulvaensis IAM 566 & $2^{3}$ & $2^{3}$ & $2^{3}$ & $2^{3}$ & $2^{4}$ & $2^{9}$ \\
\hline (37) C. zebra IAM 221 & 0 & 0 & 0 & 0 & 0 & 0 \\
\hline (38) Coelastrum astroideum & 0 & $2^{5}$ & 0 & 0 & 0 & $2^{3}$ \\
\hline (39) Eutetramorus fottii & 0 & $2^{2}$ & 0 & 0 & 0 & 0 \\
\hline (40) Monoraphidium contorfum & 0 & 0 & 0 & 0 & 0 & 0 \\
\hline (41) Scenedesmus quadricauda & $2^{6}$ & $2^{7}$ & $2^{7}$ & $2^{4}$ & $2^{5}$ & $2^{8}$ \\
\hline (42) S. ecorni & 0 & 0 & 0 & 0 & 0 & $2^{2}$ \\
\hline (43) Selenastrum capricornutum & $2^{6}$ & $2^{8}$ & $2^{7}$ & $2^{2}$ & $2^{3}$ & $2^{4}$ \\
\hline
\end{tabular}

and interpretable, but non-observable, components. Eigenvectors forming the principal components were obtained from the similarity coefficients in linear combinations of variables.
Comparison of the phylogenetic trees

The phylogenetic relationship between species of Chlorella species based on 18 SSU DNA sequence (Wu et al. 2001) 
was modified for comparison with that on haemagglutining activity of this study. A slight modification was made by deleting the species which were not used in this study. The remaining species were maintained in their original positions on the phylogenetic tree. The encoded SSU DNA sequence data in Chlorella species 21, Chlorella sp. I and Chlorella sp. V were obtained from PCR amplifications using genomic DNA in the laboratory and the identified species GenBank/EMBL databases. In addition, the NS12 region of SSU sequence from the GenBank/EMBL data base was employed. The phylogenetic tree was produced by parsimony method using of a heuristic search in PAUP 4.0 from cladistically informative characters. Values above branches are confidence levels estimated by 100 bootstrap replicates.

\section{Results}

Agglutination of human erythrocytes by cell extracts

Thirty-two of the 43 strains tested exhibited agglutinating activity against at least one of the erythrocyte types assayed (Table 1). All Chlorella cell extracts agglutinated human A, $\mathrm{B}$ and $\mathrm{O}$ erythrocytes. Of these, C. ellipsoidea, $C$. protothecoides, and C. pyrenoidosa extracts exhibited the strongest activity, reaching titres of $2^{9}-2^{11}$. Haemagglutination by Chlamydomonas was limited to 4 of 15 taxa, with an activity titre of less than $2^{5}$. Scenedesmus quadricauda and Selenastrum capricornutum extracts preferentially agglutinated human erythrocytes, while $S$. ecornis and Monoraphidium contorfum extracts failed to react with any of the erythrocyte species used. The prokaryotes Spirulina maximum and Sp. platensis also contained agglutinins that strongly agglutinated human ABO erythrocytes, while the other two species Sp. subsalsa and Synechococcus leopoliensis did not agglutinate any. However, all cell extracts giving positive reactions with human erythrocytes were non-specific, with the exception of Coelastrum astroideum, which showed anti- $(\mathrm{B}+\mathrm{H})$ activity for erythrocytes from type B blood.

Agglutination of ungulate erythrocytes by cell extracts

Fewer cell extracts were reactive against ungulate than against human erythrocytes. Twenty-four taxa exhibited agglutinating activity against cow, sheep, and pig erythrocytes; 12 of these belonged to Chlorella and 4 to Chlamydomonas species. In general, agglutinating activity was lower than for human erythrocytes. More taxa contained agglutinins that reacted with pig erythrocytes than with sheep or cow erythrocytes. The strains Chlorella sp. 21 and C. vulgaris CCAP 211/13 exhibited the strongest activity, with titres of $2^{6}-2^{8}$, against cow, sheep, and pig erythrocytes. $C$. sorokininiana exhibited stronger activity against sheep erythrocytes, and Chlorella xanthella, $C$. protothecoides, and $C$. sorokininiana exhibited stronger activity against pig erythrocytes.

\section{Statistical and multivariate analysis}

The correlations ( $r$ values) for agglutination reactions with human type A, B and O erythrocytes were higher than for cow, sheep, and pig erythrocytes (Table 1). However, all coefficients were significant $(p<0.05)$, indicating that the agglutination activity of cell extracts against the six erythrocyte types used varied together, but independently. The agglutinating characteristics of cell extracts against human erythrocytes differed from those against cow and sheep, but not pig erythrocytes (Tables 2 and 3). However, there were no significant differences in agglutination reactions between sheep and cow erythrocytes.

The result of the cluster analysis for 39 green algae is shown in Fig. 1, with two large clusters represented mainly by Chlamydomonas (C1) and Chlorella taxa (C2). Each was further divided into two subclusters with unequal numbers of taxa and distances. Subcluster 1 (Sc1) contained taxa whose extracts did not cause agglutination or that agglutinated one of ungulate or human erythrocyte species tested. Subcluster 2 (Sc2) contained taxa having agglutination activity towards two or more ungulate erythrocytes and all human erythrocytes at low titres if any. Subcluster $3(\mathrm{Sc} 3)$ contained taxa with specific activity for all human erythrocytes at high titres and one or two ungulate erythrocytes. Subcluster $4(\mathrm{Sc} 4)$ contained taxa that agglutinated all human erythrocytes tested and pig erythrocytes. Taxa in subclusters 3 and 4 contained agglutinins that preferentially agglutinated human erythrocytes, whereas those in subclusters 1 and 2 preferentially agglutinated ungulate erythrocytes. In general, the cluster-

Table 2 Correlation coefficients $(r)$ of the paired agglutination reactions of cell extracts with different erythrocytes

\begin{tabular}{lllllll}
\hline \multicolumn{2}{l}{ Erythrocyte } & & & & \multicolumn{2}{l}{$r^{*}$} \\
\hline Human & B & & & & & \\
& O & 0.930 & & & & \\
& A & 0.932 & 0.941 & & & \\
Pig & & 0.502 & 0.516 & 0.579 & & \\
Cow & & 0.442 & 0.522 & 0.478 & 0.592 & \\
Sheep & & 0.324 & 0.415 & 0.478 & 0.690 & 0.693 \\
\hline
\end{tabular}

* Significance level at least $p<0.05$.

Analysis was performed based on $\log (\mathrm{x}+1)$, where $\mathrm{x}=$ activity in titer, and $n=43$ 
Table $3 t$ values of the paired agglutination reaction of Chlorella cell extracts with different erythrocytes

\begin{tabular}{|c|c|c|c|c|c|c|c|}
\hline \multicolumn{2}{|c|}{ Erythrocyte } & \multirow{2}{*}{$\frac{\text { Mean }}{1.106}$} & \multicolumn{5}{|l|}{$t$} \\
\hline \multirow[t]{3}{*}{ Human } & B & & & & & & \\
\hline & $\mathrm{O}$ & 0.987 & 1.143 & & & & \\
\hline & A & 0.956 & 1.298 & 0.619 & & & \\
\hline \multicolumn{2}{|l|}{ Pig } & 0.899 & 1.670 & 1.310 & 1.275 & & \\
\hline \multicolumn{2}{|l|}{ Sheep } & 0.515 & $3.055^{* *}$ & $2.896^{* *}$ & $2.843^{* *}$ & $2.585^{*}$ & \\
\hline \multicolumn{2}{|l|}{ Cow } & 0.331 & $3.705 * * *$ & $3.617 * * *$ & $3.585 * * *$ & $3.371 * *$ & 2.313 \\
\hline
\end{tabular}

$*_{p}<0.05, t_{0.05(2) 76}=1.992$

$* * p<0.01, t_{0.01(2) 76}=2.642$

$* * p<0.001, t_{0.001(2) 76}=3.423$

Analysis was performed based on $\mathrm{x}=\log (\mathrm{X}+1)$, where $\mathrm{X}=$ activity in titer, and $n=43$

ing of taxa was more dependent on specificity for ungulate than for human erythrocytes. Distance between taxa in the dendrogram is inverse to similarity, thus the greater the disparity between two taxa or clusters, the greater their distance.

Principal component analysis (PCA) yielded two major components, $\mathrm{PC} 1$ and $\mathrm{PC} 2$, with eigenvalues explaining $87.7 \%$ of the total variability of reactions (Table 4). These were used to interpret the variability of haemagglutination assays. The positions of taxa in Fig. 2 reveal eigenvectors or weights on taxa on these two components. The PC1 axis expresses the activity component from taxa with higher positive eigenvectors that did not agglutinate any of the erythrocytes used. Thus, the higher value on this axis, the lower the activity found. The PC2 axis expresses the component of specificity for erythrocytes: taxa whose extracts strongly agglutinated human erythrocytes have higher positive values, and those with activity against ungulate erythrocytes have negative values.

PCA analysis separated Chlorella and Chlamydomonas taxa into two regions designated R-Clo (5-22) and R-Cla (23-37). Most Chlorella taxa lie above the PC1 axis, but Chlamydomonas lies below this axis on the positive side of the PC2 axis. This reflects the different binding specificities for human and non-human erythrocytes. The remaining algae, Coelastrum, Monoraphidium,Scenedesmus, and Selenastrum (38-43), are closely distributed on or near the PC1 axis on the positive side, in association with most of the Chlamydomonas taxa. The blue-green algae Spirulina and Synechococcus (1-4) are also located inside the Chlamydomonas region and form a small group (not shown in the figure). The arrow represents a general trend in haemagglutination characteristics from Chlorella to Chlamydomonas, with decreasing activity against human erythrocytes, but with increasing activity against ungulate erythrocytes.
Fig. 1 Dendrogram showing the clusters of 39 taxa of green microalgae. Algal species numbers refer to Table 1

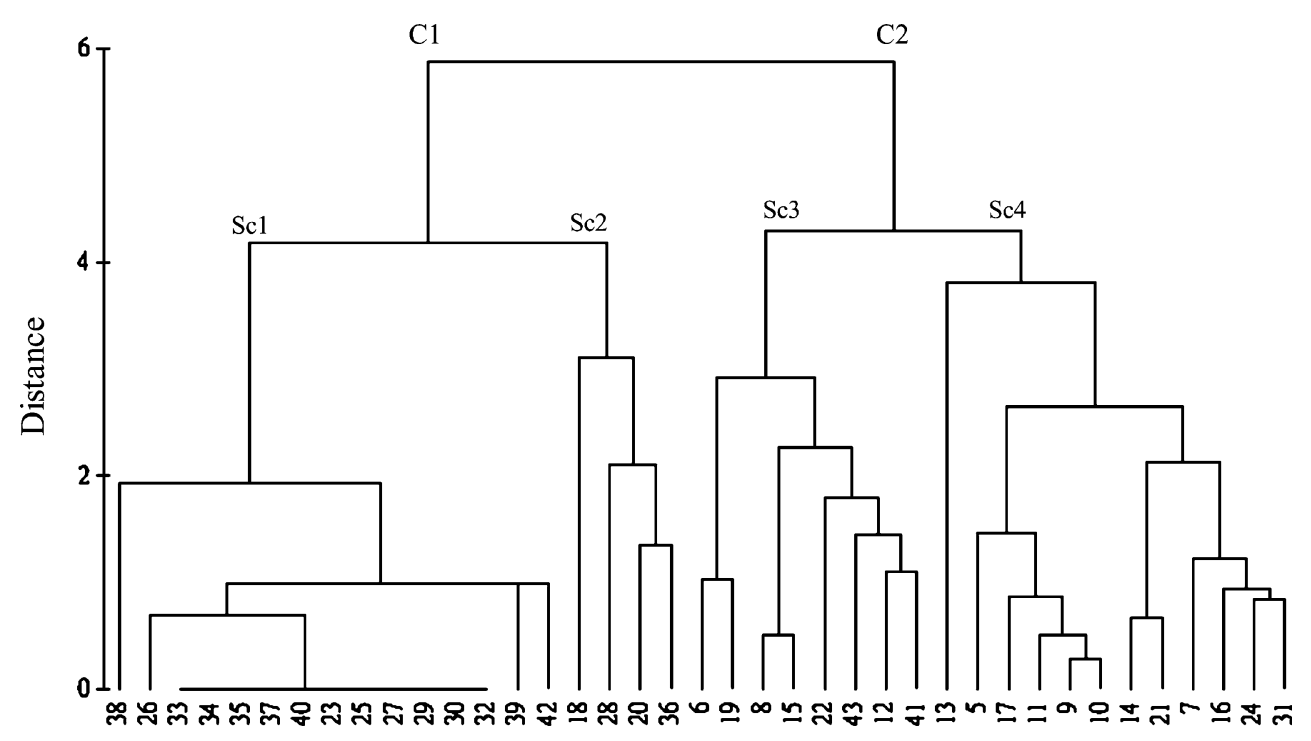

Algal species 
Table 4 Eigenvectors of the variables in the first five principal components

\begin{tabular}{|c|c|c|c|c|c|c|}
\hline \multirow[t]{2}{*}{ Variable } & & \multicolumn{5}{|c|}{ Principal component } \\
\hline & & $\mathrm{PC} 1$ & $\mathrm{PC} 2$ & $\mathrm{PC} 3$ & $\mathrm{PC} 4$ & PC5 \\
\hline \multirow[t]{3}{*}{ Human } & A & -0.451 & 0.321 & 0.079 & 0.239 & 0.006 \\
\hline & B & -0.425 & 0.434 & -0.030 & -0.129 & -0.690 \\
\hline & $\mathrm{O}$ & -0.443 & 0.352 & -0.162 & 0.030 & 0.706 \\
\hline \multicolumn{2}{|l|}{ Cow } & -0.365 & -0.451 & -0.665 & -0.436 & -0.042 \\
\hline \multicolumn{2}{|l|}{ Sheep } & -0.369 & -0.521 & 0.030 & 0.727 & -0.119 \\
\hline \multicolumn{2}{|l|}{ Pig } & -0.387 & -0.332 & 0.724 & -0.455 & 0.096 \\
\hline \multicolumn{2}{|c|}{ Cum. \% var. } & 68.8 & 87.7 & 94.3 & 98.2 & 99.3 \\
\hline
\end{tabular}

\section{Comparison on phylogenetic analysis}

After a slight modification, the phylogenetic tree (by the parsimony analysis) based on 18SSU DNA sequence of the 11 species produced two main clusters. The first cluster was further divided into two subclusters, corresponding to subcluster SC3 and SC4 in our cluster analysis based on haemagglutinating activity (Fig. 3). Species in the two subclusters were $C$. zofingiensis, C. pyrenoidosa, $C$. luteoviridis, C. saccharophila, C. ellipsodea and C. kessien, Chlorella species V. The second cluster, represented by $C$. sorokiniana, Chlorella species 21, Chlorella species I and C. vulgaris, corresponds to $\mathrm{SC} 2$ in the cluster analysis. Therefore, phylogenetic grouping of Chlorella species based on agglutinin activity agrees to a considerable extent with that based on nuclear sequence.

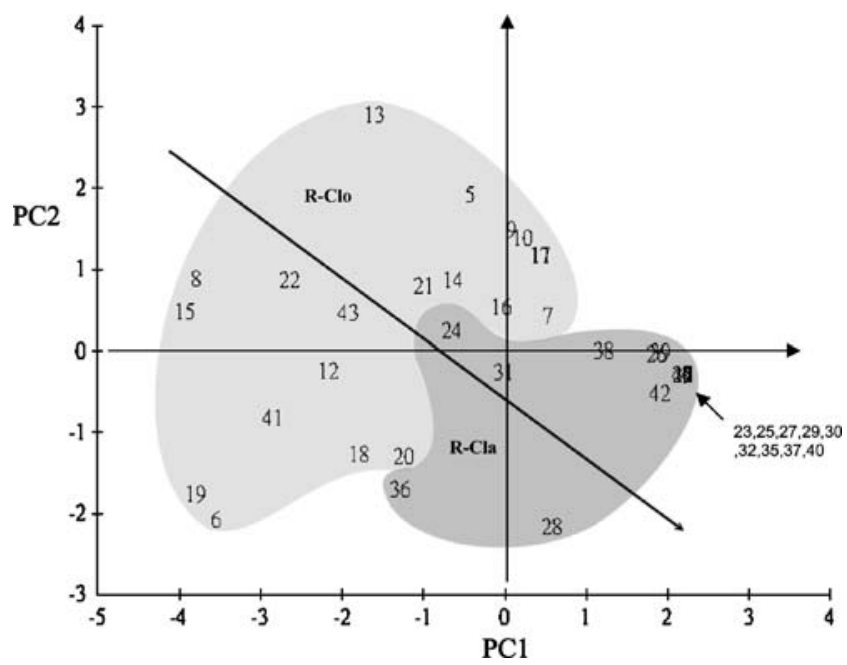

Fig. 2 Thirty-nine taxa of green microalgae distributed along two principal components based on haemagglutination characters against six types of erythrocytes. The arrow indicatese the change in haemagglutination characters with decreasing activity against human erythrocytes but with increasing activity against ungulate erythrocytes. Numbers indicate species numbers referred in Table 1

\section{Discussion}

Most of the microalgae studied here contained agglutinins capable of agglutinating at least one type of mammalian erythrocyte. All Chlorella extracts preferentially agglutinated human erythrocytes, whereas haemagglutination by Chlamydomonas extracts was limited to fewer species and to lesser degrees. Nevertheless, all the algae showing positive reaction towards human erythrocytes contained non-specific agglutinins for $\mathrm{ABH}$ antigens, with the exception of Coelastrum astroideum, which had anti- $(\mathrm{B}+\mathrm{H})$ specificity. Such activity has been reported previously for the marine algae Ptilota plumosa (Rogers et al. 1977) and Bryopsis hypnoides (Hori et al. 1981).

Sheep and rabbit erythrocytes have been commonly used for the detection of haemagglutinating activity from seaweeds (Hori et al. 1981; Fabregas et al. 1985; Freitas et al. 1997). However, human erythrocytes are superior to sheep erythrocytes for this purpose in evaluating extracts of marine microalgae (Chu et al. 2005; Liao and Huang 2000; Liao et al. 2001). Our assays confirmed this. In addition, pig erythrocytes appeared to be more sensitive than cow and sheep erythrocytes in the agglutination assay.

Haemagglutinating activity has some taxonomic significance (Rogers and Fish 1991), as it exhibits great variability between species, as demonstrated in seaweeds from various ecological habitats (Blunden et al. 1978; Fabregas et al. 1985; Freitas et al. 1997). However, the assumption was never given with statistical analysis of the variability. Thus, we are interested in what the variability is between species in relation to specificity for antigens or carbohydrate receptors on erythrocytes, and whether the statistical analysis of haemagglutinating activity would show some taxonomic pattern. Multivariate analysis tools, such as principal component analysis or cluster analysis, have been widely used to interpret and group the variability of morphometric measurements of various organisms as well as in genetics and immunology (Blackith and Reyment 1971). In the present study, the erythrocyte types assayed could be used as the determining variables in clustering or grouping of the algae, provided the within-assay variations were small in comparison with those between taxa or erythrocyte types. Statistical analysis showed that the agglutination reactions of algal extracts with all the erythrocytes tested were related. Furthermore, agglutination reactions with pig erythrocytes did not differ significantly from those with human erythrocytes, but did differ from cow and sheep erythrocytes $(p<0.05)$. This suggests that pig erythrocytes share more common antigens with human erythrocytes than those of other two ungulates, by approximately $7-10 \%$ (estimated from $t$ values in the two-tailed distribution table; Zar 1974). Thus, the variability of agglutinating activity and 


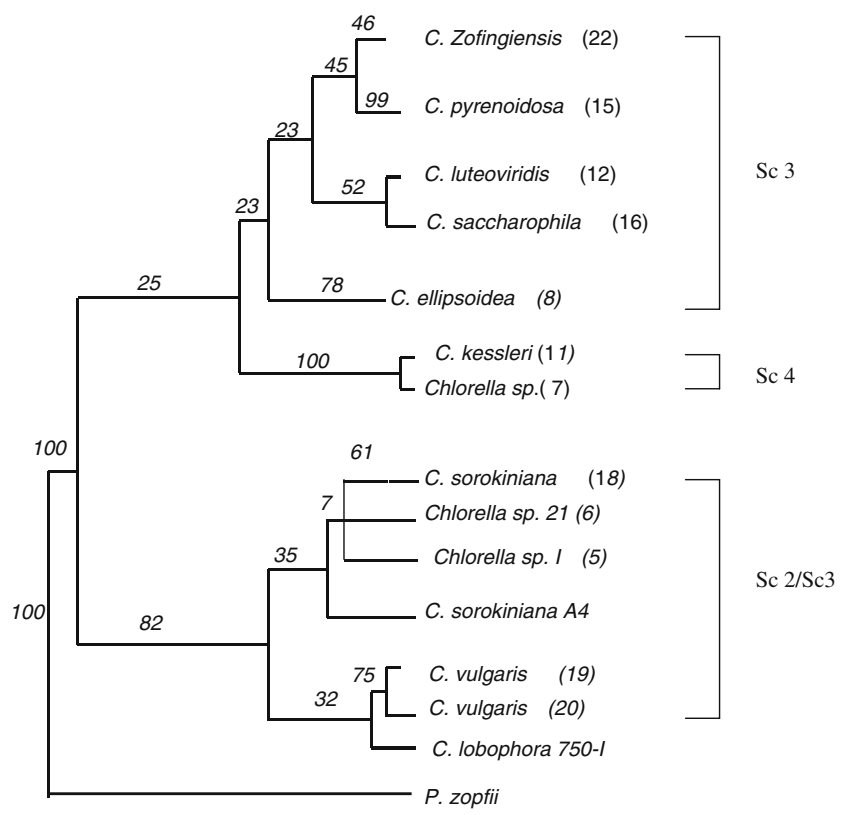

Fig. 3 Phylogenetic relationships inferred by Chlorella nuclear SSU NS12 nucleotide sequence data and modified from Wu et al. (2001). SC2, SC3, and SC4 are corresponding subclusters based on haemagglutining activities

the subsequent clustering or grouping of taxa by multivariate analyses may have been due mainly to the presence of anti-pig agglutinins in algal extracts.

Cluster analysis revealed four subclusters of taxa with significant differences in agglutination patterns, which were not observable when considering individual species. Principal component analysis produced a two-dimensional interpretation. This proved satisfactory in distinguishing the haemagglutination characteristics of Chlamydomonas extracts from those of Chlorella extracts. Similarities and dissimilarities among and between taxa are evident from their relative positions on the two components in this analysis. For example, C. fusca and its variety C. fusca var. vacuolata, closely placed in Fig. 2, exhibited identical haemagglutination reactions. By contrast, the two strains C. vulgaris-CCAP and C. vulgaris-NIES appear in the same region as Chlorella although they were widely separated in two subclusters in the dendrogram (Fig. 1). Thus, principal component analysis can provide complementary information on agglutinin characteristics that is not shown by cluster analysis. It is not surprising that Scenedesmus quadricauda and $S$. econis are seen as separate, as they have extremely different haemagglutination characteristics. Whether this separation is of taxonomic relevance is not known. By contrast, the four prokaryotes in Synechococcus and Spirulina species lie within the Chlamydomonas region, suggesting that their binding specificities overlap with those of Chlamydomonas cells.
It appeared that the clustering or grouping of Chlorella species based on haemagglutinating activities was similar to results with grouping based on $18 \mathrm{~S}$ rDNA sequences (Wu et al. 2001).Thus, we propose that agglutinating characteristics can be used in taxonomy (see also Blunden et al. 1978).

In this study, we used native or untreated mammalian erythrocytes. Treatment of erythrocytes to improve the agglutination assay could cause a bias in analysis and thus distort the true haemagglutination variability in the assayed algae. Proteases such as pronase, trypsin, and papain have been used to improve the detection of agglutinins in some marine algae (Rogers and Fish 1991; Freitas et al. 1997; Liao and Huang 2000, Liao et al. 2001). Additionally, the presence of haemolysin, which masks haemagglutination reactions in the extracts, can also distort analysis (Rogers et al. 1980; Hori et al. 1981). Nevertheless, the well-defined haemagglutination characteristics of Chlorella and Chlamydomonas extracts seen in this study suggest that agglutinins in microalgae have evolved through changes in binding specificity, and that these have genetic significance.

Recently, we have purified algal agglutins, CPH (Chlorella pyreniodosa hemagglutinin; $58.0 \mathrm{kDa}$ ) and $\mathrm{CLH}$ (Chlorella lutevirtidis hemagglutinin; $58.3 \mathrm{kDa}$ ), from $C$. pyreniodosa and $C$. luteovirtidis, respectively, and found those algal lectins possess similar properties (Chu et al. 2006). We have also developed specific monoclonal antibodies for $\mathrm{CPH}$ and CPL. Immunogold labeling and electron microscopy revealed that both $\mathrm{CPH}$ and $\mathrm{CPL}$ are localized beneath the surface layer of the cell, between the cytoplasm and cell membrane. Furthermore, western blotting revealed that above antibodies recognized each microalga of sc3 clearly (unpublished data). However, microalgae in both sc2 and sc3 were not easily recognized by those antibodies. Those results also support our hypothesis that algal agglutining activity could be an important taxonomic character.

The multivariate analysis used here gives useful information and is useful in the interpretation of haemagglutination variability between algal extracts, particularly when many data are available from screening studies. Several reports indicate that microalgae contain agglutinins that agglutininate human and animal erythrocytes to varying degrees (Hori et al. 1996; Liao and Huang 2000; Sato et al. 2000). Some agglutinins have shown pronounced antitumor effects, such as a glycoprotein from Chlorella vulgaris (Tanaka et al. 1998). They also exhibit unique structure and characteristics, as shown in extracts from Microcystis aeruginosa, M. viridis, Oscillatoria agardhii and related species (Sakamoto et al. 1996; Yamaguchi et al. 1998, 1999; Sato et al. 2000). Thus, microalgae may prove to be a good source of diverse agglutinins for biochemical studies or commercial applications. 
Acknowledgements We thank the National Science Council of the Republic of China (Taiwan) for supporting this study (NSC: 92-2317B-002-030). We also thank Mr. Chen Yua-Sin (Good Biotech Co.) for providing erythrocytes for agglutination assay.

\section{References}

Blackith RE, Reyment RA (1971) Multivariate morphometrics. Academic Press, London

Blunden G, Rogers DJ, Farnham WF (1978) Haemagglutinins in British marine algae and their possible taxonomic value. In: Irvine DE, Price JH (eds) Modern approaches to the taxomomy of red and brown algae. Academic Press, London, pp 21-45

Boyd WC, Almodovar LR, Boyd LG (1966) Agglutinins in marine algae for human erythrocytes. Transfusion 6:82-83

Brown RM, John CJ, Bold HC (1968) Electron and phase-contrast microscopy of sexual reproduction in Chlamydomonas moewusii. J Phycol 4:100-120

Carmichael WW, Bent PE (1981) Haemagglutination method for detection of freshwater cyanobacteria (blue-green algae) toxins. Appl Env Microbiol 41:1383-1388

Chu CY, Liao WR, Lin LP (2005) Haemagglutinating activities from unicellular algae. Acta Ocenanogr Taiwanica 42:15-23

Chu CY, Huang R, Lin LP (2006) Purification and Characterization of a Novel Haemagglutinin from Chlorella pyrenoidosa. J Ind Microbiol Biotechnol 33:967-973

Fabregas J, Llovo J, Munoz A (1985) Haemagglutinins in red seaweeds. Bot Mar 28:517-520

Freitas ALP, Teixeira DIA, Costa FHF, Farias WRL, Lobato ASC, Sampaio AH, Benevides NMB (1997) A new survey of Brazilian marine algae for agglutinins. J Appl Phycol 9:495-501

Hori K, Miyazawa K, Ito K (1981) Haemagglutinins in marine algae. Bull Jap Soc Sci Fish 47:793-798

Hori K, Takehiko O, Hisao K, Mimuro M (1996) Lectin-like and lectin receptors in marine microalgae: Haemagglutination and reactivity with purified lectins. J Phycol 32:783-790

Illman AM, Scragg AH, Shales SW (2000) Increase in Chlorella strains calorific values when grown in low nitrogen medium. Enz Microbial Technol 27:631-635
Liao WR, Huang R (2000) Agglutination of human and animal erythrocytes in marine unicellular algae. J Ind Microbiol Biotechnol 24:262-266

Liao WR, Huang R, Su HM (2001) Haemagglutinating activity from marine microalgae. Nova Hedwigia Bei 122:99-106

Rogers DJ, Blunden G, Evans PR (1977) Ptilota plumosa, a new source of a blood-group B specific lectin. Med Lab Sci 34:193-200

Rogers DJ, Fish BC (1991) Marine algal lectins. In Kilpatrick DC, Van Driessche E, Bog-Hansen TC (eds), Lectin review. Sigma Chemical, St. Louis, pp 129-142

Rogers DJ, Blunden G, Topliss JA, Guiry MD (1980) A survey of some marine organisms for haemagglutinins. Bot Mar 23:569-577

Sato Y, Murakami M, Miyazawa K, Hori K (2000) Purification and characterization of a novel lectin from a freshwater cyanobacterium, Oscillatoria agardhii. Comp Biochem Physiol B 125: $169-177$

Saito T, Matsuda Y (1984) Sexual agglutinin of mating-typing minus gametes in Chlamydomonas reinhardii. II. Purification and characterization of minus agglutinin and comparison with plus agglutinin. Arch Microbiol 139:95-99

Sakamoto S, Yamaguchi M, Watanabe MF, Watanabe M, Kamiya H (1996) Distribution and characterization of lectins from natural and cultured Microcystis spp. In: Yasumoto T, Oshima Y, Fukuyo Y (eds). Harmful and Toxic Algal Blooms, Inter-Government Oceanographic Commission of UNESCO, pp 569-572

Tanaka K, Yamada A, Noda K (1998) A novel glycoprotein obtained from Chlorella vulgaris strain CK22 shows antimetastatic immunopotentiation. Canc Immunol Immu 45:313-320

Wu HL, Hseu RS, Lin LP (2001) Identification of Chlorella spp. isolates using ribosomal DNA sequences. Bot Bull Acad Sin 42:115-121

Yamaguchi M, Mituru J, Ryuichi S, Koji M, Kamiya H (1998) Purification and characterization of Microcysis aeruginosa (freshwater cyanobacterium) lectin. Comp Biochem Physiol B 119:593-597

Yamaguchi M, Ogawa T, Muramoto K, Kamio Y, Jimbo M, Kamiya H (1999) Isolation and characterization of a mannanbinding lectin from the freshwater cyanobacterium (blue-green algae) Microcystis viridis. Biochem Biophys Res Commun 265:703-708

Zar JH (1974) Biostatistical analysis. Prentice-Hall, EnglewoodCliffs, N.J. 